\title{
Utilization of amino acid analogues in diets of young turkeys*
}

\author{
By C. M. PARSONSt AND L. M. POTTER \\ Department of Poultry Science, Virginia Polytechnic Institute and State University, \\ Blacksburg, Virginia 24061 USA
}

(Received 19 November 1980 - Accepted 12 January 1981)

1. The relative potencies of three lysine, one tryptophan and six methionine analogues to their corresponding $\mathrm{L}$-amino acids were determined.

2. Male poults, $9 \mathrm{~d}$ of age, were used in five $14 \mathrm{~d}$ experiments. Experimental diets were formed by adding increasing levels of an amino acid (L-isomer) or its analogues to a basal diet deficient in the test amino acid. Multiple-regression analyses of body-weight gain $v$. level of added or amount of consumed amino acid supplement were computed. Relative potencies of the analogues were calculated using the slope-ratio technique.

3. Type of independent 'ariable used in the regression analyses did not significantly affect relative potency values.

4. From regression analyses with level of added amino acid supplement as the independent variable, relative potencies (with $95 \%$ confidence intervals) on a molar basis of each analogue as a percentage of the respective amino acid were: hydroxymethyl-L-tryptophan monohydrate $9(-12,22)$, DL-methionine sulphone $3(-68,36)$. DL-methionine hydantoin - $10(-63,29)$, L-cystine $8(-31,40)$, DL-methionine sulphoxide $59(24,95)$ and 46 $(18,74)$, oleoyl-DL-methioni:e $77(37,138)$, methionine hydroxy analogue calcium $93(65,128)$, dicocoyl-L-lysine $23(5,41)$, bis-hydroxymethyl-L-lysine-calcium $69(54,82)$ and $41(-1,80)$, mono-hydroxymethyl-L-lysine-calcium $89(77,101)$ and $99(65,134)$.

5. The first four analognes appeared to have little or no amino acid activity. DL-methionine sulphoxide, dicocoyl-L-lysine, and bis-hydroxymethyl-L-lysine-calcium were partially active. Relative potencies of oleoylDL-methionine, methionine hydroxy analogue calcium, and mono-hydroxymethyl-L-lysine-calcium were not significantly different from those of their corresponding amino acids.

Methionine, lysine and tryptophan are generally considered to be the three most limiting amino acids in most commercial poultry and livestock rations. DL-methionine and methionine hydroxy analogue calcium (MHAC), and L-lysine hydrochloride have been used successfully as sources of methionine and lysine respectively in poultry rations. No economical substitute for tryptophan is available at present. Additional research is needed to obtain analogues of methionine, lysine and tryptophan that provide successful substitutes for these amino acids in animal rations.

Recent studies have indicated that amino acid analogues which are protected against rumen microbial degradation may offer potential for ruminant nutrition (Chalupa, 1975; Buttery et al. 1977). Postrumen administration of protein has increased performance of lactating dairy cows, whereas responses to postrumen administration of individual amino acids have been inconsistent (Clark, 1975). The relative potencies of several protected amino acid analogues were cletermined in this study as the young turkey provides a convenient and economical experimental animal for determining utilization of such analogues under comparable condition:s postruminally.

In certain types of tood processing, methionine present in food proteins may undergo oxidation to methionine sulphoxide or methionine sulphone (Anderson et al. 1976). Although methionine sulphone is reported to have no methionine activity in chicks and rats, results concerning methionine sulphoxide are inconsistent (Kuzmicky et al. 1977). The

* Portions of a thesis prisented by the senior author in partial fulfillment of the requirements for the degree of Master of Science at Vir zinia Polytechnic Institute and State University.

+ Current address: Department of Animal Science, 332 Mumford Hall, 1301 W. Gregory Dr., University of Illinois, Urbana, Illinois 61301 USA. 
Table 1. Composition $(\mathrm{g} / \mathrm{kg})$ of basal diets

\begin{tabular}{|c|c|c|c|c|}
\hline \multirow[b]{2}{*}{ Ingredients } & \multicolumn{4}{|c|}{ Expt no. } \\
\hline & 1 & 2 & 3 & $4-5$ \\
\hline Ground yellow maize & 467.6 & $465 \cdot 5$ & $485 \cdot 4$ & $329 \cdot 7$ \\
\hline Glucose monohydrate* & $20 \cdot 0$ & $10 \cdot 0$ & $60 \cdot 0$ & $135 \cdot 1$ \\
\hline $\begin{array}{l}\text { Hydrolyzed animal and } \\
\text { vegetable fat } \\
\text { Maize-gluten meal }\end{array}$ & $20 \cdot 0$ & $20 \cdot 0$ & - & $20 \cdot 0$ \\
\hline$(600 \mathrm{~g}$ protein $/ \mathrm{kg})$ & $380 \cdot 0$ & $200 \cdot 0$ & $150 \cdot 0$ & - \\
\hline Dehulled soya-bean meal & - & $200 \cdot 0$ & $150 \cdot 0$ & $373 \cdot 0$ \\
\hline Menhaden fish meal & $50 \cdot 0$ & $50 \cdot 0$ & $20 \cdot 0$ & $30 \cdot 0$ \\
\hline Gelatin & - & - & $80 \cdot 0$ & $70 \cdot 0$ \\
\hline Mono and dicalcium phosphate & $25 \cdot 0$ & $25 \cdot 0$ & - & - \\
\hline Defiuorinated phosphate & - & - & $35 \cdot 0$ & $35 \cdot 0$ \\
\hline Ground limestone & $20 \cdot 0$ & $20 \cdot 0$ & - & - \\
\hline Iodized salt & $4 \cdot 0$ & $4 \cdot 0$ & $4 \cdot 0$ & $4 \cdot 0$ \\
\hline Trace mineral mix ${ }^{\dagger}$ & 0.5 & 0.5 & 0.5 & 0.5 \\
\hline Vitamins and food additives & 1.9 & 1.9 & 1.9 & 1.9 \\
\hline DL-methionine & - & 0.4 & $2 \cdot 1$ & - \\
\hline L-arginine & 8.0 & $2 \cdot 2$ & - & - \\
\hline L-histidine & - & - & 0.5 & 0.5 \\
\hline L-isoleucine & - & - & $1 \cdot 2$ & - \\
\hline L-lysine hydrochloride & - & - & $7 \cdot 3$ & 0.2 \\
\hline L-threonine & $2 \cdot 0$ & 0.2 & $1 \cdot 7$ & $0 \cdot 1$ \\
\hline L-tryptophan & $1 \cdot 0$ & 0.3 & - & - \\
\hline L-valine & - & - & 0.4 & - \\
\hline Calculated analysis $(\mathrm{g} / \mathrm{kg}) \S$ & & & & \\
\hline Energy $(\mathrm{kJ} \mathrm{ME} / \mathrm{kg})$ & $13,810 \cdot 5$ & $12,647 \cdot 1$ & $12,655 \cdot 4$ & $12,052.8$ \\
\hline Protein & $310 \cdot 0$ & 292.0 & $291 \cdot 0$ & $290 \cdot 0$ \\
\hline Lysine & $7 \cdot 4$ & $11 \cdot 9$ & - & 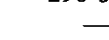 \\
\hline Tryptophan & - & - & $2 \cdot 0$ & - \\
\hline Methionine & - & - & - & $4 \cdot 4$ \\
\hline Cystine & - & - & - & 3.4 \\
\hline
\end{tabular}

ME, Metabolizable energy.

- Cerelose obtained from Corn Products Company, Englewood Cliffs, NJ.

+ Supplied (mg/kg diet): 185 manganese sulphate, 70 zinc oxide, 167 ferric citrate, 20 cupric sulphate, 1 cobalt acetate, 30 potassium iodate, 9 sodium molybdate, 0.438 sodium selenite.

† Supplied $/ \mathrm{kg}$ diet: $3.78 \mathrm{mg}$ retinol acetate, $82.5 \mu \mathrm{g}$ cholecalciferol, $11 \mathrm{mg}$ DL- $x$-tocopheryl acetate, $7 \mathrm{mg}$ menadione sodium bisulphite complex, $1.1 \mathrm{mg}$ thiamine hydrochloride, $4.4 \mathrm{mg}$ riboflavin, $11.0 \mathrm{mg}$ calcium D-pantothenate, $44 \mathrm{mg}$ niacin, $500 \mathrm{mg}$ choline chloride, $13.2 \mu \mathrm{g}$ cyanocobalamin, $1.65 \mathrm{mg}$ folic acid, $55 \mu \mathrm{g}$ biotin, $1.1 \mathrm{mg}$ pyridoxine hydrochloride, $125 \mathrm{mg}$ ethoxyquin, $44 \mathrm{mg}$ bacitracin.

$\$$ From amino acid analysis, basal diets used in the experiments contained the following (g/kg): Expt 1, 6.4 lysine; Expt 2, 12.6 lysine; Expt 4, 4.6 methionine, 2.8 cystine; Expt 5, 5.0 methionine, 3.4 cystine. Sulphur amino acids determined using the performic acid procedure of Moore (1963).

availability of methionine sulphoxide and methionine sulphone for the young turkey may be important since most commercial diets contain proteins that have undergone processing which may have resulted in oxidation of methionine. Therefore, the relative potencies of methionine sulphoxide and methionine sulphone for the young turkey were determined in the present study.

It is apparent from the review of Harms et al. (1976) that much controversy still exists concerning the relative potencies of DL-methionine and MHAC for poultry. Thus, additional information concerning the biological availability of these methionine sources was obtained.

Recently, Behrends \& Waibel (1980) reported that the L-cystine replacement value for 
Table 2. Chemical structures of some analogues tested

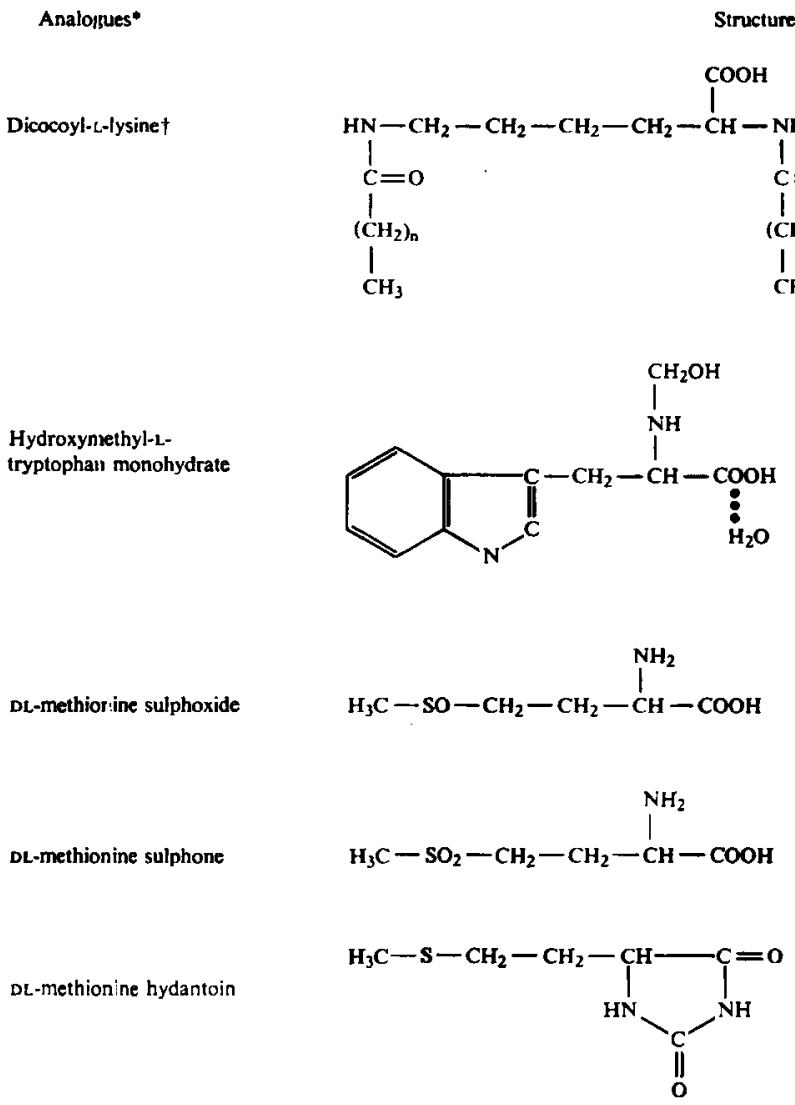

* Supplied by Degussa, Inc., Teterboro, New Jersey. Mono-hydroxymethyl-L-lysine-calcium, bishydroxymethyl-L-lysine-calcium, and oleoyl-DL-methionine were also supplied by Degussa, Inc.

$\dagger n 6$ or 8 (a mixture).

starting poults is $55 \%$ of the total sulphur amino acid requirement. Therefore, the relative potency of L-cystine to L-methionine was also determined in a test diet deficient in total $\mathrm{S}$ amino acids.

\section{MATERIALS AND METHODS}

Five experiments involving medium-size male turkeys were conducted. The males were fed on a practical-type starter diet from 1 to $9 \mathrm{~d}$ of age. At that time, the poults were assigned according to weight into pens containing eight poults each so that the average body-weights of the groups were similar.

The basal diets used in this study were formulated to contain at least $110 \%$ of the (US) National Research Counil (1977) recommended levels of all essential amino acids, except the amino acid under test, for poults 1-4 weeks of age (Table 1). Chemical structures of mono-hydroxymethyl-L-lysine-calcium (MHLC) and bis-hydroxymethyl-L-lysine-calcium (BHLC) were published by Parsons et al. (1980) and oleoyl-DL-methionine by Buttery et al. (1977), while the chemical structures of other analogues tested are presented in Table 
2. Elemental analysis of MHLC, BHLC and hydroxymethyl-L-tryptophan monohydrate (HTM) produced the following values:

MHLC: $\mathrm{C}_{14} \mathrm{H}_{30} \mathrm{O}_{6} \mathrm{~N}_{4} \mathrm{Ca}$

Calculated: C 43.1, H 7.7, N 14.3, Ca 10.3

Found: $\mathrm{C} 39 \cdot 9, \mathrm{H} 6 \cdot 5, \mathrm{~N} 12 \cdot 4, \mathrm{Ca} 12 \cdot 9$

BHLC: $\mathrm{C}_{16} \mathrm{H}_{34} \mathrm{O}_{8} \mathrm{~N}_{4} \mathrm{Ca}$

Calculated: $\mathrm{C} 42 \cdot 7, \mathrm{H} 7 \cdot 6, \mathrm{~N} 12 \cdot 4$, Ca $8 \cdot 9$

Found: C 42.6, $\mathrm{H} 7 \cdot 0, \mathrm{~N} 12 \cdot 9$, Ca $9 \cdot 9$

$\mathrm{HTM}: \mathrm{C}_{11} \mathrm{H}_{16} \mathrm{O}_{4} \mathrm{~N}_{2}$

Calculated: $\mathrm{C} 57 \cdot 1, \mathrm{H} 6 \cdot 3, \mathrm{~N} 11 \cdot 1$

Found: C 60.2, H 6.0, N $10 \cdot 9$

Analytical values for MHLC indicated approximately $90 \%$ purity. Elemental analysis of BHLC and HTM were in accordance with theoretical composition. Nuclear magnetic resonance analyses of dicocoyl-L-lysine, DL-methionine sulphoxide, DL-methionine sulphone, DL-methionine hydantoin and oleoyl-DL-methionine were in accordance with theoretical composition.

Experimental diets were formed by substituting for glucose monohydrate in the basal diet with increasing levels of the test amino acid (L-isomer) or an analogue. A practical maize-soya-bean meal diet (Parsons et al. 1980) was also included as a positive control. Experimental diets were fed for a period of $14 \mathrm{~d}$.

Poults were housed in thermostatically controlled Petersime batteries and food and water were supplied $a d$ lib. Body-weight gains and food consumptions were measured at $7 \mathrm{~d}$ intervals.

Multiple regression analyses were calculated using average body-weight gains (9-23 d of age) as the dependent variable and level of dietary addition or amount of amino acid supplement consumed as the independent variable. Relative potencies of the amino acid analogues with $95 \%$ confidence limits were calculated using the slope-ratio technique of Finney (1971).

\section{RESULTS}

\section{Expt 1}

The relative potencies of MHLC, BHLC and dicocoyl-L-lysine to L-lysine were determined. Supplementation of the basal diet with increasing levels of L-lysine, MHLC or BHLC resulted in linear increases in average body-weight gains and food efficiencies (Table 3). The relative potency of MHLC was approximately $90 \%$ on a molar basis and not significantly different from that of L-lysine. BHLC was partially active but the relative potency of the analogue was significantly less than that of MHLC or L-lysine. The relative potency of dicocoyl-L-lysine was not significantly different from zero. Average body-weight gains of poults fed on the basal diet supplemented with the highest level of L-lysine were substantially less than those of poults fed on the practical-type diet $(131 v .364 \mathrm{~g})$.

\section{Expt 2}

Relative potencies of MHLC and BHLC were determined using a basal diet which contained less maize-gluten meal than the one used in Expt 1. A linear improvement in growth performance was obtained from adding increasing levels of L-lysine, MHLC and BHLC to the basal diet (Table 4). As observed in Expt 1, the relative potency of MHLC was not significantly different from L-lysine on a molar basis. BHLC was approximately $45 \%$ active on a molar basis. Average body-weight gain of poults fed on the basal diet supplemented with $5 \mathrm{~g} \mathrm{~L}$-lysine/ $\mathrm{kg}$ was $253 \mathrm{~g}$ as compared to $263 \mathrm{~g}$ for poults fed on the practical-type diet. 
Table 3. Expt 1. Growth performance from 9 to $23 d$ of age and relative potencies of lysine analogues

(Values for diets containing supplemental lysine represent the average of two groups containing eight male poults each. All other values represent the average of one group containing eight male poults. Average body-weight gain and food efficiency of two groups of poults fed on the practical-type diet were $364 \mathrm{~g}$ and 0.699 , respectively. Upper and lower $95 \%$ confidence limits given in parentheses)

\begin{tabular}{|c|c|c|c|c|c|c|c|}
\hline \multirow{3}{*}{$\begin{array}{l}\text { Amino acid } \\
\text { supplement }\end{array}$} & \multirow{3}{*}{$\begin{array}{c}\text { Dietary } \\
\text { addition } \\
(\mathrm{g} / \mathrm{kg})\end{array}$} & \multirow{3}{*}{$\begin{array}{c}\text { Body-wt } \\
\text { gain(g) }\end{array}$} & \multirow{3}{*}{$\begin{array}{c}\text { Food } \\
\text { efficiency }\end{array}$} & \multicolumn{4}{|c|}{ Relative potency* } \\
\hline & & & & \multicolumn{2}{|c|}{ Wt basis } & \multicolumn{2}{|c|}{ Molar basis } \\
\hline & & & & Addition & Consumption & Addition & Consumption \\
\hline L-lysine & $\begin{array}{l}0.0 \\
2 \cdot 0 \\
4 \cdot 0 \\
6 \cdot 0 \\
8 \cdot 0\end{array}$ & $\begin{array}{r}23.8 \\
39.4 \\
67.6 \\
105.8 \\
130.4\end{array}$ & $\begin{array}{l}0.194 \\
0.283 \\
0.388 \\
0.478 \\
0.553\end{array}$ & $100 \cdot 0$ & $100-0$ & $100 \cdot 0$ & $100 \cdot 0$ \\
\hline $\begin{array}{l}\text { Mono-hydroxy- } \\
\text { methyl-L-lysine- } \\
\text { calcium }\end{array}$ & $\begin{array}{l}2 \cdot 0 \\
4 \cdot 0 \\
6 \cdot 0 \\
8 \cdot 0\end{array}$ & $\begin{array}{l}42.7 \\
53 \cdot 2 \\
74.8 \\
89.4\end{array}$ & $\begin{array}{l}0.308 \\
0.364 \\
0.435 \\
0.488\end{array}$ & $\begin{array}{c}66.1 \\
(57,75)\end{array}$ & $\begin{array}{c}80 \cdot 2 \\
(71,90)\end{array}$ & $\begin{array}{c}88.7 \\
(77,101)\end{array}$ & $\begin{array}{c}107.4 \\
(95.120)\end{array}$ \\
\hline $\begin{array}{l}\text { Bis-hydroxy- } \\
\text { methyl-L-lysine- } \\
\text { calcium }\end{array}$ & $\begin{array}{l}2.0 \\
4.0 \\
6.0 \\
8.0\end{array}$ & $\begin{array}{l}36 \cdot 7 \\
36 \cdot 2 \\
57 \cdot 5 \\
66 \cdot 4\end{array}$ & $\begin{array}{l}0.264 \\
0.268 \\
0.358 \\
0.377\end{array}$ & $\begin{array}{c}44 \cdot 0 \\
(35,53)\end{array}$ & $\begin{array}{c}52 \cdot 8 \\
(43,63)\end{array}$ & $\begin{array}{c}68.1 \\
(54.82)\end{array}$ & $\begin{array}{c}81 \cdot 7 \\
(66,97)\end{array}$ \\
\hline $\begin{array}{l}\text { Dicocoyl- } \\
\text { L-lysine }\end{array}$ & $\begin{array}{r}6.0 \\
12.0 \\
18.0 \\
24 \cdot 0\end{array}$ & $\begin{array}{l}18 \cdot 3 \\
20 \cdot 7 \\
27 \cdot 5 \\
35 \cdot 2\end{array}$ & $\begin{array}{l}0.156 \\
0.163 \\
0.266 \\
0.277\end{array}$ & $\begin{array}{r}4 \cdot 1 \\
(1,7)\end{array}$ & $\begin{array}{c}2.9 \\
(-2.8)\end{array}$ & $\begin{array}{c}22.8 \\
(5 ; 41)\end{array}$ & $\begin{array}{c}16 \cdot 0 \\
(-10.42)\end{array}$ \\
\hline
\end{tabular}

- Relative potencies from thes slope-ratio assay were calculated using level of dietary addition or amount of supplement consumed as the independent variable.

\section{Expt 3}

The relative potency of HTM to L-tryptophan was determined. Supplementation of the basal diet with 0.2 and $0.4 \mathrm{~g} \mathrm{~L}$-tryptophan $/ \mathrm{kg}$ resulted in a linear increase in growth performance (Table 5). Little or no growth response was obtained from HTM, and the relative potency of this analogue was not significantly different from zero. Average body-weight gain of poults fed on the basal diet containing $0.4 \mathrm{~g}$ added L-tryptophan $/ \mathrm{kg}$ was 269 as compared to $317 \mathrm{~g}$ for poults fed on a practical-type diet.

\section{Expt 4}

The potencies of DL-methionine sulphoxide, DL-methionine sulphone, DL-methionine hydantoin and oleoyl-DL-methionine (OM) were compared to L-methionine. A linear improvement in growth ferformance was obtained from addition of increasing levels of L-methionine to the basal diet (Table 6). DL-methionine sulphoxide was approximately 50\% active, and the relative potency of this analogue was significantly less than $100 \%$. Little or no growth response was obtained from DL-methionine sulphone or DL-methionine hydantoin, and the relative potencies of these analogues were not significantly different from zero. Although a linear increase in growth performance was obtained from the first three added levels of $\mathrm{OM}$, growth of poults at the $10 \mathrm{~g} / \mathrm{kg}$ level was less than expected. The relative potency of OM on a molar basis was approximately $110 \%$ as calculated from the first three 
Table 4. Expt 2. Growth performance from 9 to $23 d$ of age and relative potencies of lysine analogues

(Values represent the average of two groups containing eight male poults each. Average body-weight gain and food efficiency of two groups of poults fed on the practical-type diet were $262.6 \mathrm{~g}$ and 0.630 , respectively. Upper and lower $95 \%$ confidence limits given in parentheses)

\begin{tabular}{|c|c|c|c|c|c|c|c|}
\hline \multirow{3}{*}{$\begin{array}{l}\text { Amino acid } \\
\text { supplement }\end{array}$} & \multirow{3}{*}{$\begin{array}{c}\text { Dietary } \\
\text { addition } \\
(\mathrm{g} / \mathrm{kg})\end{array}$} & \multirow{3}{*}{$\begin{array}{c}\text { Body-wt } \\
\text { gain }(g)\end{array}$} & \multirow{3}{*}{$\begin{array}{l}\text { Food } \\
\text { efficiency }\end{array}$} & \multicolumn{4}{|c|}{ Relative potency* } \\
\hline & & & & \multicolumn{2}{|c|}{ Wt basis } & \multicolumn{2}{|c|}{ Molar basis } \\
\hline & & & & Addition & Consumption & Addition & Consumption \\
\hline L-lysine & $\begin{array}{l}0.0 \\
1.0 \\
2.0 \\
3.0 \\
4.0 \\
5.0\end{array}$ & $\begin{array}{l}182 \cdot 2 \\
218 \cdot 3 \\
219 \cdot 8 \\
222 \cdot 7 \\
238 \cdot 4 \\
253 \cdot 4\end{array}$ & $\begin{array}{l}0.584 \\
0.651 \\
0.655 \\
0.668 \\
0.674 \\
0.687\end{array}$ & $100 \cdot 0$ & $100 \cdot 0$ & $100 \cdot 0$ & $100 \cdot 0$ \\
\hline $\begin{array}{l}\text { Mono-hydroxy- } \\
\text { methyl-L-lysine- } \\
\text { calcium }\end{array}$ & $\begin{array}{l}1 \cdot 0 \\
2 \cdot 0 \\
3 \cdot 0 \\
4 \cdot 0\end{array}$ & $\begin{array}{l}175 \cdot 2 \\
197 \cdot 8 \\
213 \cdot 3 \\
232 \cdot 2\end{array}$ & $\begin{array}{l}0.601 \\
0.623 \\
0.629 \\
0.664\end{array}$ & $\begin{array}{c}73 \cdot 9 \\
(48,101)\end{array}$ & $\begin{array}{c}77 \cdot 8 \\
(52,105)\end{array}$ & $\begin{array}{c}99 \cdot 2 \\
(65,134)\end{array}$ & $\begin{array}{c}103 \cdot 4 \\
(69,141)\end{array}$ \\
\hline $\begin{array}{l}\text { Bis-hydroxy- } \\
\text { methyl-L-lysine- } \\
\text { calcium }\end{array}$ & $\begin{array}{l}1 \cdot 0 \\
2 \cdot 0 \\
3 \cdot 0 \\
4 \cdot 0\end{array}$ & $\begin{array}{l}171 \cdot 5 \\
188 \cdot 6 \\
185.9 \\
203 \cdot 2\end{array}$ & $\begin{array}{l}0.585 \\
0.607 \\
0.613 \\
0.635\end{array}$ & $\begin{array}{c}26 \cdot 5 \\
(-4,53)\end{array}$ & $\begin{array}{c}28 \cdot 8 \\
(-1,80)\end{array}$ & $\begin{array}{c}41 \cdot 1 \\
(-1,80)\end{array}$ & $\begin{array}{c}44 \cdot 5 \\
(-7,88)\end{array}$ \\
\hline
\end{tabular}

- Relative potencies from the slope-ratio assay were calculated using level of dietary addition or amount of supplement consumed as the independent variable.

Table 5. Expt 3. Growth performance from 9 to $23 \mathrm{~d}$ of age and relative potency of $a$ tryptophan analogue

(Values represent the average of two groups containing eight male poults each. Average body-weight gain and food efficiency of two groups fed on the practical-type diet were $317.0 \mathrm{~g}$ and 0.658 , respectively. Upper and lower $95 \%$ confidence limits given in parentheses)

\begin{tabular}{|c|c|c|c|c|c|}
\hline \multirow{2}{*}{$\begin{array}{l}\text { Amino acid } \\
\text { supplement }\end{array}$} & \multirow{2}{*}{$\begin{array}{c}\text { Dietary } \\
\text { addition } \\
(\mathrm{g} / \mathrm{kg})\end{array}$} & \multirow{2}{*}{$\begin{array}{l}\text { Body-wt } \\
\text { gain }(g)\end{array}$} & \multirow{2}{*}{$\begin{array}{l}\text { Food } \\
\text { efficiency }\end{array}$} & \multicolumn{2}{|c|}{$\begin{array}{l}\text { Relative potency* } \\
\text { on a molar basis }\end{array}$} \\
\hline & & & & Addition & Consumption \\
\hline L-tryptophan & $\begin{array}{l}0.00 \\
0.20 \\
0.40\end{array}$ & $\begin{array}{l}174 \cdot 2 \\
224 \cdot 8 \\
269 \cdot 4\end{array}$ & $\begin{array}{l}0.565 \\
0.658 \\
0.680\end{array}$ & $100 \cdot 0$ & $100 \cdot 0$ \\
\hline $\begin{array}{l}\text { Hydroxymethyl } \\
\text { L-tryptophan } \\
\text { monohydrate }\end{array}$ & $\begin{array}{l}0.25 \\
0.49 \\
0.74\end{array}$ & $\begin{array}{l}189 \cdot 7 \\
183 \cdot 8 \\
190 \cdot 8\end{array}$ & $\begin{array}{l}0.641 \\
0.599 \\
0.581\end{array}$ & $\begin{array}{c}9 \cdot 3 \\
(-12,22)\end{array}$ & $\begin{array}{c}7.6 \\
(-14,22)\end{array}$ \\
\hline
\end{tabular}

* Relative potencies from the slope-ratio assay were calculated using level of dietary addition or amount of supplement consumed as the independent variable. 


\section{Table 6. Expt 4. Growth performance from 9 to $23 d$ of age and relative potencies of methionine analogues}

(Values for oleoyl-DL-methionine represent the average of one group containing eight male poults. All other values represent the average of two groups containing eight male poults each. Average body-weight gain and food efficiency of two groups fed on the practical-type diet were $247 \cdot 1 \mathrm{~g}$ and $0 \cdot 593$, respectively. Upper and lower $95 \%$ confidence limits given in parentheses)

\begin{tabular}{|c|c|c|c|c|c|c|c|}
\hline \multirow{3}{*}{$\begin{array}{l}\text { Amino acid } \\
\text { supplement }\end{array}$} & \multirow{3}{*}{$\begin{array}{c}\text { Dietary } \\
\text { addition } \\
(\mathrm{g} / \mathrm{kg})\end{array}$} & \multirow{3}{*}{$\begin{array}{l}\text { Body-wt } \\
\text { gain }(g)\end{array}$} & \multirow{3}{*}{$\begin{array}{c}\text { Food } \\
\text { efficiency }\end{array}$} & \multicolumn{4}{|c|}{ Relative potency* } \\
\hline & & & & \multicolumn{2}{|c|}{ Wt basis } & \multicolumn{2}{|c|}{ Molar basis } \\
\hline & & & & Addition & Consumption & Addition & Consumption \\
\hline L-methionine & $\begin{array}{l}0.0 \\
0.5 \\
1.0 \\
1.5 \\
2.0\end{array}$ & $\begin{array}{l}165 \cdot 1 \\
193 \cdot 8 \\
197 \cdot 8 \\
228 \cdot 3 \\
233 \cdot 6\end{array}$ & $\begin{array}{l}0.531 \\
0.550 \\
0.561 \\
0.592 \\
0.609\end{array}$ & $100 \cdot 0$ & $100-0$ & $100 \cdot 0$ & $100 \cdot 0$ \\
\hline $\begin{array}{l}\text { DL-methionine } \\
\text { sulphoxide }\end{array}$ & $\begin{array}{l}0.5 \\
1.0 \\
1.5 \\
2.0\end{array}$ & $\begin{array}{l}196.2 \\
191.4 \\
196.7 \\
206 \cdot 1\end{array}$ & $\begin{array}{l}0.572 \\
0.575 \\
0.576 \\
0.597\end{array}$ & $\begin{array}{c}53 \cdot 3 \\
(22,83)\end{array}$ & $\begin{array}{c}62 \cdot 0 \\
(29,98)\end{array}$ & $\begin{array}{c}59 \cdot 0 \\
(24,95)\end{array}$ & $\begin{array}{c}68 \cdot 8 \\
(32,108)\end{array}$ \\
\hline $\begin{array}{l}\text { DL-methionine } \\
\text { sulphone }\end{array}$ & $\begin{array}{l}0.5 \\
1.0 \\
1.5\end{array}$ & $\begin{array}{l}178 \cdot 2 \\
166 \cdot 0 \\
181 \cdot 4\end{array}$ & $\begin{array}{l}0.547 \\
0.551 \\
0.545\end{array}$ & $\begin{array}{c}2 \cdot 8 \\
(-56,46)\end{array}$ & $\begin{array}{c}8 \cdot 8 \\
(-52,56)\end{array}$ & $\begin{array}{c}3 \cdot 4 \\
(-68,36)\end{array}$ & $\begin{array}{c}10 \cdot 7 \\
(-57,61)\end{array}$ \\
\hline $\begin{array}{l}\text { DL-methionine } \\
\text { hydantoin }\end{array}$ & $\begin{array}{l}0.5 \\
1.0 \\
1.5 \\
2.0\end{array}$ & $\begin{array}{l}180 \cdot 4 \\
160 \cdot 3 \\
155 \cdot 4 \\
184 \cdot 9\end{array}$ & $\begin{array}{l}0.552 \\
0.525 \\
0.499 \\
0.545\end{array}$ & $\begin{array}{c}-8 \cdot 8 \\
(-55,23)\end{array}$ & $\begin{array}{c}-2.4 \\
(-49,32)\end{array}$ & $\begin{array}{c}-10 \cdot 4 \\
(-63,29)\end{array}$ & $\begin{array}{c}-2 \cdot 8 \\
(-60,35)\end{array}$ \\
\hline $\begin{array}{l}\text { Oleoyl-DL- } \\
\text { methionine }\end{array}$ & $\begin{array}{r}2.5 \\
5.0 \\
7.5 \\
10.0\end{array}$ & $\begin{array}{l}185 \cdot 3 \\
211 \cdot 1 \\
227 \cdot 5 \\
202 \cdot 9\end{array}$ & $\begin{array}{l}0.546 \\
0.583 \\
0.600 \\
0.584\end{array}$ & $\begin{array}{r}15 \cdot 4 \\
(8,25)\end{array}$ & $\begin{array}{r}16 \cdot 5 \\
(9,26)\end{array}$ & $\begin{array}{c}76.8 \\
(37,138)\end{array}$ & $\begin{array}{c}82.5 \\
(43,130)\end{array}$ \\
\hline
\end{tabular}

- Relative potencies from the slope-ratio assay were calculated using level of dietary addition or amount of supplement consumed as the independent variable.

levels of addition only ard approximately $80 \%$ when calculated from all four levels. These values were not significantly different from $100 \%$. Growth performance obtained from the basal diet supplemented with $2 \mathrm{~g} \mathrm{~L}$-methionine/ $\mathrm{kg}$ was slightly less than that obtained from the practical-type diet (234v247 g).

\section{Expt 5}

The potencies of DL-methionine, MHAC, DL-methionine sulphoxide and L-cystine were compared to L-methionine. The relative potencies of DL-methionine and MHAC were approximately 107 and $9.3 \%$, respectively (Table 7). The differences between DL-methionine or MHAC and L-methionine were not significant. DL-methionine sulphoxide was approximately $45 \%$ active. The relative potency of L-cystine was not significantly different from zero. Growth performance of poults fed on the basal diet supplemented with $2 \mathrm{~g}$ $\mathrm{L}$-methionine $/ \mathrm{kg}$ was similar to that obtained from the practical-type diet (250 v. $246 \mathrm{~g}$ ).

\section{DISCUSSION}

Of the amino acid analogues tested in this study, MHLC, BHLC, dicocoyl-L-lysine, HTM and $\mathrm{OM}$ are examples of protected amino acid analogues that may have a potential use in ruminant nutrition. The relative potency of $\mathrm{MHLC}$ was not significantly different from 
Table 7. Expt 5. Growth performance from 9 to $23 d$ of age and relative potencies of methionine analogues

(Values for L-cystine represent the average of one group containing eight male poults. All other values represent the average of two groups containing eight male poults each. Average body-weight gain and food efficiency of two groups fed on the practical-type diet were $246.1 \mathrm{~g}$ and 0.694 , respectively. Upper and lower $95 \%$ confidence limits given in parentheses)

\begin{tabular}{|c|c|c|c|c|c|c|c|}
\hline \multirow{3}{*}{$\begin{array}{l}\text { Amino acid } \\
\text { supplement }\end{array}$} & \multirow{3}{*}{$\begin{array}{l}\text { Dietary } \\
\text { addition } \\
(\mathrm{g} / \mathrm{kg})\end{array}$} & \multirow{3}{*}{$\begin{array}{l}\text { Body-wt } \\
\text { gain (g) }\end{array}$} & \multirow{3}{*}{$\begin{array}{l}\text { Food } \\
\text { efficiency }\end{array}$} & \multicolumn{4}{|c|}{ Relative potency* } \\
\hline & & & & \multicolumn{2}{|c|}{ Wt basis } & \multicolumn{2}{|c|}{ Molar basis } \\
\hline & & & & Addition & Consumption & Addition & Consumption \\
\hline L-methionine & $\begin{array}{l}0.0 \\
0.5 \\
1.0 \\
1.5 \\
2.0\end{array}$ & $\begin{array}{l}179 \cdot 3 \\
213 \cdot 4 \\
217 \cdot 0 \\
239 \cdot 9 \\
249 \cdot 7\end{array}$ & $\begin{array}{l}0.584 \\
0.631 \\
0.643 \\
0.663 \\
0.667\end{array}$ & $100 \cdot 0$ & $100 \cdot 0$ & $100 \cdot 0$ & $100 \cdot 0$ \\
\hline $\begin{array}{r}\text { DL-methionine } \\
\quad \cdots\end{array}$ & $\begin{array}{l}0.51 \\
1.01 \\
1.52 \\
2.02\end{array}$ & $\begin{array}{l}212 \cdot 4 \\
225 \cdot 5 \\
232 \cdot 9 \\
258 \cdot 0\end{array}$ & $\begin{array}{l}0.617 \\
0.640 \\
0.660 \\
0.701\end{array}$ & $\begin{array}{c}106 \cdot 7 \\
(80,143)\end{array}$ & $\begin{array}{c}106 \cdot 2 \\
(82,139)\end{array}$ & $\begin{array}{c}106 \cdot 7 \\
(80,143)\end{array}$ & $\begin{array}{c}106 \cdot 2 \\
(82,139)\end{array}$ \\
\hline $\begin{array}{l}\text { Methionine } \\
\text { hydroxy ana- } \\
\text { logue calcium } †\end{array}$ & $\begin{array}{l}0.54 \\
1.08 \\
1.61 \\
2.15\end{array}$ & $\begin{array}{l}212 \cdot 8 \\
215 \cdot 2 \\
225 \cdot 7 \\
242 \cdot 5\end{array}$ & $\begin{array}{l}0.622 \\
0.626 \\
0.632 \\
0.659\end{array}$ & $\begin{array}{c}82 \cdot 1 \\
(58,112)\end{array}$ & $\begin{array}{c}81 \cdot 6 \\
(59,110)\end{array}$ & $\begin{array}{c}93 \cdot 2 \\
(65,128)\end{array}$ & $\begin{array}{c}92 \cdot 5 \\
(67,125)\end{array}$ \\
\hline $\begin{array}{l}\text { DL-methionine } \\
\text { sulphoxide }\end{array}$ & $\begin{array}{l}0.5 \\
1.0 \\
1.5 \\
2.0\end{array}$ & $\begin{array}{l}202 \cdot 7 \\
220-5 \\
221-2 \\
203 \cdot 4\end{array}$ & $\begin{array}{l}0.628 \\
0.633 \\
0.645 \\
0.633\end{array}$ & $\begin{array}{c}42 \cdot 0 \\
(16,67)\end{array}$ & $\begin{array}{c}46 \cdot 6 \\
(21,72)\end{array}$ & $\begin{array}{c}46 \cdot 3 \\
(18,74)\end{array}$ & $\begin{array}{c}51 \cdot 6 \\
(24,80)\end{array}$ \\
\hline L-cystine & $\begin{array}{l}0.5 \\
1.0 \\
1.5 \\
2.0\end{array}$ & $\begin{array}{l}192 \cdot 7 \\
188 \cdot 0 \\
206 \cdot 4 \\
197 \cdot 2\end{array}$ & $\begin{array}{l}0.617 \\
0.611 \\
0.609 \\
0.609\end{array}$ & $\begin{array}{c}7 \cdot 7 \\
(-31,40)\end{array}$ & $\begin{array}{c}9 \cdot 3 \\
(-28,42)\end{array}$ & $\begin{array}{c}7 \cdot 7 \\
(-31,40)\end{array}$ & $\begin{array}{c}9 \cdot 3 \\
(-28,42)\end{array}$ \\
\hline
\end{tabular}

* Relative potencies from the slope-ratio assay were calculated using level of dietary addition or amount of supplement consumed as the independent variable.

+ DL-methionine $(98 \%)$ pure, Degussa, Inc.; methionine hydroxy analogue calcium $(93 \%)$ pure, Monsanto Industrial Chemicals $\mathrm{Co}$.

that of L-lysine in both experiments in which it was tested. Parsons et al. (1980) also reported that MHLC had substantial lysine activity in diets of young turkeys. A weighted mean for the potency of MHLC from the present study and that of Parsons et al. (1980) was calculated by the method of Finney (1971) to be $82 \%$. This extent of lysine activity indicates that MHLC is utilized efficiently as a source of lysine by the young turkey. Buttery et al. (1977) found that a similar type of methionine derivative, $N$-hydroxymethyl-DL-methionine-calcium, could replace L-methionine in chick diets. The latter investigators also reported that $N$-hydroxymethyl-DL-methionine-calcium was resistant to rumen microbial degradation in sheep and concluded that this analogue fulfilled the criteria for a potential source of methionine in ruminant diets. The efficient utilization of MHLC by young turkeys suggests that this analogue may also be a potential source of lysine for ruminants. However, the extent of post rumen utilization of amino acid analogues in the ruminant may differ substantially from the extent of utilization in the turkey.

In contrast to MHLC, the relative potency of BHLC was significantly less than that of 
L-lysine. Results of previous studies with $\epsilon-N$ and $\alpha-N$ acetyl (Neuberger \& Sanger, 1943) and $\epsilon-N$ and $\alpha-N$ methyl (Gordon, 1939) derivatives of L-lysine indicated that $\epsilon-N$ derivatives of L-lysine were available for growth of rats, but $\alpha-N$ derivatives were not available. However, in the present study, the $\epsilon-N$ hydroxymethyl group of BHLC appeared to be the reason for the lower relative potency of BHLC as compared to MHLC since both derivatives contained $\alpha-N$ hydroxymethyl groups.

Dicocoyl-L-lysine had little biological activity for replacing L-lysine in diets of young turkeys. Stephens $e$ al. (1977) also found that $\epsilon-N$ and $\alpha-N$ monopalmitoyl derivatives of L-lysine and $\alpha-N, \epsilon-N$ dipalmitoyl-L-lysine had no lysine activity in rats. These results suggest that long-chain acyl derivatives of $\mathrm{L}$-lysine are not utilized efficiently for growth in single stomached animals.

HTM had little or no tryptophan activity. Evidently, the $\alpha-N$ hydroxymethyl group renders this compound unavailable for replacing L-tryptophan in diets of young turkeys.

The relative potency of $\mathrm{OM}$ was not significantly different from that of $\mathrm{L}$-methionine. Buttery et al. (1977) also found that OM could replace methionine in chick diets and that this analogue was resistant to rumen microbial degradation in sheep. Substantial hydrolysis of OM was also obtained from in vitro incubation with kidney acylase (Damico, 1975). Results of the present study indicated that $O M$ is utilized efficiently as a source of methionine by young turkeys and this analogue may have potential as a source of methionine for ruminants.

The negative potency value obtained for DL-methionine hydantoin indicates that this analogue cannot replace methionine in diets of young turkeys. Damico (1975) also concluded that DL-methionine hydantoin could not be used as a source of supplemental methionine for human foods.

DL-methionine sulphone had no methionine activity for young turkeys. This observation is in agreement with previous studies with rats and chicks (Kuzmicky et al. 1977). Therefore, oxidation of methionine to methionine sulphone in food proteins during processing would render the methionine unavailable for growth.

DL-methionine sulphoxice was approximately $50 \%$ active as compared to L-methionine. This observation is in general agreement with some studies and in contrast to higher potency values obtained in other studies as discussed by Kuzmicky et al. (1977) and Gjøen \& Njaa (1977). Kuzmicky et al. (1977) reported that L-methionine sulphoxide was more available in chick diets than D- and DL-methionine sulphoxide and suggested that differences in activity values among studies were due to the stereoisomeric form of methionine sulphoxide used. $\mathrm{Njaa}(1962)$ also found that $\mathrm{D}-, \mathrm{DL}$ - and L-methionine sulphoxide were 50,75 and $100 \%$ as active as DL-methionine, respectively, for rats. Further studies of the biological availability of methionine sulphoxide should primarily concern the L-form of the analogue since oxidation of methionine in food protein would result in formation of L-methionine sulphoxide.

The relative potency of MHAC was slightly less but not significantly different from that of L- or DL-methionine on a molar basis. These results are in agreement with most previous studies which have employed practical diets containing intact proteins (Harms $e t$ al. 1976). However, in general, these studies have not been sensitive enough to provide precise estimations of the reative potency of MHAC. Results of studies employing purified or semi-purified diets have consistently indicated that the biological activity of MHAC is less than that of L-or DL-methionine (Katz \& Baker, 1975). More precise values for the biological activity of MHAC as compared to L- and DL-methionine in practical diets are needed.

Little or no linear growth response was obtained from adding L-cystine to a maize-soyabean meal-gelatin diet. This diet contained $4.4 \mathrm{~g}$ methionine and $3.4 \mathrm{~g}$ crystine $/ \mathrm{kg}$ from 
calculation. Evidently, the basal diet was not sufficiently deficient in cystine to provide a significant linear increase in growth for the levels of added L-cystine.

The type of independent variable, either level of dietary addition or amount of amino acid supplement consumed, used in the regression analyses had no significant effect on relative potency values. Thus, both methods of calculation of relative potency values appear satisfactory.

Supplementation of the basal diets used in Expts 2-5 with the respective limiting amino acids resulted in growth performance approaching that obtained from the practical-type diet. Further supplementation of these diets with the respective test amino acids produced growth equal to that obtained from a practical-type diet (Parsons, 1978). In contrast, growth of poults fed on the high-maize-gluten-meal diet in Expt 1 supplemented with $8 \mathrm{~g} \mathrm{L-lysine/kg}$ was much less than growth of poults fed on the practical-type diet. Parsons \& Potter (1979) reported that supplementation of this high-maize-glutten-meal diet with isoleucine and valine or choline chloride or both in the presence of 0-20 $\mathrm{g}$ added L-lysine $/ \mathrm{kg}$ failed to improve body-weight gains. The reason for the poor growth obtained from the high-maizegluten-meal diet is unknown at present.

This study was supported in part by a grant-in-aid from Degussa, Inc., Teterboro, NJ. Several of the analogues used in the experiments were supplied by R. Fahnenstich, Degussa, Inc., Teterboro, NJ.

\section{REFERENCES}

Anderson, G. H., Ashley, D. V. M. \& Jones, J. D. (1976). J. Nutr. 106, 1108.

Behrends, B. R. \& Waibel, P. E. (1980). Poult. Sci. 59, 849.

Buttery, P. J., Manomai-Udom, S. \& Lewis, D. (1977). J. Sci. Fd Agric. 28, 481.

Chalupa, W. (1975). J. Dairy Sci. 58, 1198.

Clark, J. H. (1975). J. Dairy Sci. 58, 1178.

Damico, R. (1975). J. Agric. Fd Chem. 23, 30.

Finney, D. J. (1971). Statistical Method of Biological Assay, 2nd ed. New York: Hafner.

Gjøen, A. U. \& Njaa, L. R. (1977). J. Nutr. 37, 93.

Gordon, W. G. (1939). J. biol. Chem. 127, 487.

Harms, R. H., Eldred, A. R. \& Damron, B. L. (1976). Poult. Sci. 55, 1794.

Katz, R. S. \& Baker, D. H. (1975). Poult. Sci. 54, 584.

Kuzmicky, D. D., Kohler, G. O., Walker, H. G., Jr. \& Mackey, B. E. (1977). Poult. Sci. 56, 1560.

Moore, S. (1963). J. biol. Chem. 238, 235.

National Research Council (1977). Nutrient Requirements of Domestic Animals. vol. 1. Nutrient Requirements of Poultry. Washington, D.C.: National Academy of Sciences.

Neuberger, N. \& Sanger, F. (1943). Biochem. J. 37, 515.

Njaa, L. R. (1962). Br. J. Nutr. 16, 571.

Parsons, C. M. (1978). Utilization of amino acid analogues and determination of amino acid requirements in diets of young turkeys. MS Thesis, Virginia Polytechnic Institute and State University.

Parsons, C. M. \& Potter, L. M. (1979). Poult. Sci. 58, 1091.

Parsons, C. M., Potter, L. M. \& Shelton, J. R. (1980). Poult. Sci. 59, 1852.

Stephens, C. A., Veen-Baigent, J. J., Paquet, A. \& Anderson, G. H. (1977). Can. J. Physiol. Pharmacol. 55, 434. 